\title{
Recall and Recognition of User Codes: PIP versus PIN Personal Identification Code
}

\author{
Karl W Sandberg ${ }^{1 *}$, Yan Pan ${ }^{2}$ and Trevor Archer ${ }^{3}$
}

${ }^{1}$ Dalarna University, School of Technology and Business Studies, Sweden

${ }^{2}$ Service Consulting Ltd, Sweden

${ }^{3}$ University of Gothenburg, Department of Psychology, Sweden

\begin{abstract}
A Personal Identification Number (PIN) can be used to authenticate the user to a system. PINs are often used for automated teller machines (ATMs), computer, mobile phones smart phones, the point of sale, for debit cards and credit cards. The aim of the present study was to investigate whether it was easier to remember the personal identification code composed of picture (PIP-code) than the traditional personal identification code composed of number (PIN-code). The study consists of two experiments: In Experiment 1, the PIP-code was compared with PINcode in cued recall test of the assigned codes, and in Experiment 2, the PIP-code was compared with the PIN-code in recognition tests of the assigned codes and backward recall of the cue. Each experiment was completed with both an immediate and a delayed test. The findings were relatively straightforward: First, PIP-codes were easier to recall and recognize than PIN-codes. Second, as expected recognition was superior to recall for both types of codes. The present results are discussed in the context of user-friendly procedures that enhance material to-be-remembered.
\end{abstract}

Keywords: Personal identity code; PIP; PIN; Recall; Recognition

\section{Introduction}

Personal identity involves the unique numerical identity of individuals through time. It presupposes the necessary and sufficient conditions under which an individual at one time and an individual at another time may referred to as the same person, persisting through time [1]. The concept of personal identity offers the notion concerning the diachronic, 'happening over time', problem of personal identity. The synchronic, a predicate function with one or more parameters (usually time or location), problem is grounded in the question of what features or traits characterize a given person at one time. In the derivation of personal identity codes (such as PIN-codes) the notion of identity emerges with particular meaningfulness.

\section{Pictures as Personal Identification Code?}

People with the facility of bank credit cards tend to prefer Automatic Teller Machines (ATMs) rather than go to banks during business hours. However, the cost of this innovation is that the technical items require protection with security procedures that are less sophisticated than their data processing counterparts. The reasons are twofold. First, the systems are almost entirely guarded by numeric codes, for example, the current popular four digits Personal Identification Number (PIN). It has proved to be a difficult task when users possess several codes simultaneously thereby leading to confusion/mix-up. The second reason is that the PIN codes rely heavily on effective memory performance.

When users express dissatisfaction with their assigned PIN-codes, it is probably due to an experienced irrational relationship between the code and its orientated information. The concept of meaningfulness ecompasses the notion of identities and the notion of a biographical life [2]. It refers to those attributes of a code that lead the user to readily associate the code with the item, object, instruction, or action that the code represents [3]. This argument was advocated by Bartlett [4] as question the Ebbinghaus tradition, which had dominated psychology for 50 years. Bartlett [4] argued that the study of nonsense syllable learning had excluded the most central and characteristic feature of human memory by excluding meaning.
It is likely that the meaningfulness of codes (memorablility) may be necessary to current enterprises with the growing emphasis on user friendliness. Based on this consideration, the present study proposes a novel notion of the Personal Identification Picture code (PIP-code), which refers to codes that consist of combinations of pictures.

Accordingly, the details of pictures observed may be explained also within the frameworks of cognitive neuropsychological theories $[5,6]$. The pictorial superiority effect provides the source of inspiration for the framework of the pictorial codes [7-9]. Taking into account the multitude of problems that are confronted on PIN-codes, the need for new research that bring to light more user-friendly codes [10].

\section{Code Use}

Codes are expected to present a shortened way of representing longer messages, and for that reason it is desirable to allow each code convey as much information as possible. There are two broad types of codes; arbitrary and mnemonic. Arbitrary codes aim to provide unique identification with little or no special significance to the user (e.g. PINcodes). Mnemonic codes are designed purposely to convey information that has some meaningfulness to users and tend to be easier to recall than arbitrary codes. An individual's name is a mnemonic code and represents a shorthand way of referring to a person without descriptions of age, height, weight, etc. When codes function as input media to provide access to the different application systems, two kinds of errors may occur: clerical or procedural errors. Generally, clerical errors relate

*Corresponding author: Karl W Sandberg, Dalarna University, School of Technology and Business Studies, Sweden, Tel: +4660148588; Fax: +4660148830; E-mail: karl.w.sandberg@miun.se

Received: October 10, 2018; Accepted: October 25, 2018; Published: November 01, 2018

Citation: Sandberg KW, Pan Y, Archer T (2018) Recall and Recognition of User Codes: PIP versus PIN Personal Identification Code. Clin Exp Psycho 4: 200. doi: 10.4172/2471-2701.1000200

Copyright: @ 2018 Sandberg KW, et al. This is an open-access article distributed under the terms of the Creative Commons Attribution License, which permits unrestricted use, distribution, and reproduction in any medium, provided the original author and source are credited 
to code design and occur due to faulty processing at one of three major cognitive stages: perceptual, intellectual, or movement control [11]. A procedural error occurs when a user selects and enters the wrong code in the right location or the right code in the wrong location. However, the most costly errors may be those not detected and eventually result in a general lack of integrity for the detected system.

\section{Long-term visual memory}

Visual imagery represents a powerful memory aid in Long Term Memory (LTM). Previous imagery research may be traced back to the 1800s [12]. There are two areas of prominence: first, the strong relationship between the imaginative ability of a word and its memorability; and second, the central role of imagery in mnemonic strategies. In the 1960s, the notion that remembering words plus images is better than words alone is similar to leaving two notes than one emerged. Possibly due to the circumstance that pictures are inherently more memorable than words; pictures are coded both visually and verbally, not always the case with words. Thus, one may utilize this possibility by visualizing to-be-remembered material.

Interest in long-term visual memory instigated much research. Nickerson [7] observed limitless capacity to remember pictures even if only viewed for merely a few seconds. Shepard [8] found that subjects were $98 \%$ correct on the immediate testing of 68 old-new pairs following the study of 600 pictures. Moreover, subjects were more accurate at remembering pictures than words $(90 \%)$ or sentences $(88 \%)$ tested by the same method. Standing [13] found that the performance was even remarkably high following a single presentation of each of 10,000 different pictures. Initially, pictures of faces always led to high levels of recognition memory, with no sign of forgetting over a 48-hour interval [14]. These observations underline the influence of "meaningfulness" on the storage of visual patterns.

\section{Conceptual model of the PIP-code}

In the development of new sets of codes, Field [15] has suggested construction. First, an established code should address the following issues: the coded information, the purpose for use, the required media appearance, and the accuracy requirements, including error detection and correction. Second, the codes are constructed so that users through knowledge of construction rules may easily determine code meaning and even construct new codes if necessary. Third, codes must be designed for the least skilled users within the expected population of code users. Fourth, codes need to be 'meaning-associated' yet avoid auditory or visual similarity.

It is known from experimental memory studies that pictures are easier to remember than verbal material. The present purpose was whether or not this evidence may be generalized to practical application in the field of cognitive ergonomics; e.g. using pictures instead of digits, to remember Personal Identification Codes.

Suppose one was asked to remember one hundred different pictures presented randomly. One may be familiar with some of them, but the chance of being able to name all the pictures is minimal. If prepared to remember systematically, most individuals would prefer to first classify the pictures into several categories, such as human beings, animals, vehicles, sports, buildings, landscapes and others. Later, differences among the same category of pictures would be sought. Finally, organization into a certain order would render the pictures meaningful and easier to remember. Conscious organization facilitates retrieval. Furthermore, the value of organization applies not only numeric and verbal material but also pictorial material. Objects that individuals organize into meaningful, coherent pictures were remembered better than objects in a jumbled picture [16].

\section{Code Story}

The PIP-code model defined a story pattern composed of three basic conceptual components: Subject, Object or Place/Time (SOP/T). With these elements, one may construct a simple one-sentence story. For example, Susan $(\mathrm{S})$ read a book $(\mathrm{O})$ in the library $(\mathrm{P})$. Subsequently it was decided that PIP-code should include at least three different pictures oriented to this one-sentence-story-grammar method. The first one should represent a picture of a human being, the subject. The second one should represent a picture of an object. The third one should represent a picture of a place/time, for example, an umbrella can show a rainy day. This arrangement is exemplified in Figure 1.

\section{Construction of a code story}

With the three conceptual elements, SOP/T, already in hand, the construction of a story may proceed. A simple method was to present opportunities for free-association. Whereas the elements of SOP/T were well-known pictures, individuals' semantic knowledge was similar to each other [17]. Neverthess, associations arising from the pictures may construe expressions of episodic knowledge, a particular event with personal experiences. The events individuals experience differ; what is remember most effective has emotional content. The materials, story pictures (person, thing and building or landmark) may be regarded as "noun pictures" to borrow the term from grammatical language. A different verb may associate the "noun pictures" decided by individuals' episodic knowledge. For instance, it is irrelevant whether the story was "Einstein was playing the violin at Princeton" or "Einstein was listening to a violin concerto at home" from the pictures from Figure 1. The ultimate point pertains to the facilitatory effects of the story through the subjects remember the picture codes; associations bred by the story were of minor interest. In general, subjects were encouraged to use their imagination to make the story vivid, in accordance with personal preferences by the method of "free-association" of the conceptual elements in SOP/T.

\section{Aim of the study}

The PIP-code notion was brought into the investigation in order to ensure the following aims: First, development of a user-friendly code composed of pictures called the PIP-code, that should accomplish the heuristic requirements of cognitive ergonomics design of codes; Second, to investigate whether or not the PIP-code facilitated remembering more effectively than the traditional PIN-code. These aims were tested in two experiments. In Experiment 1, the PIP-code was compared to

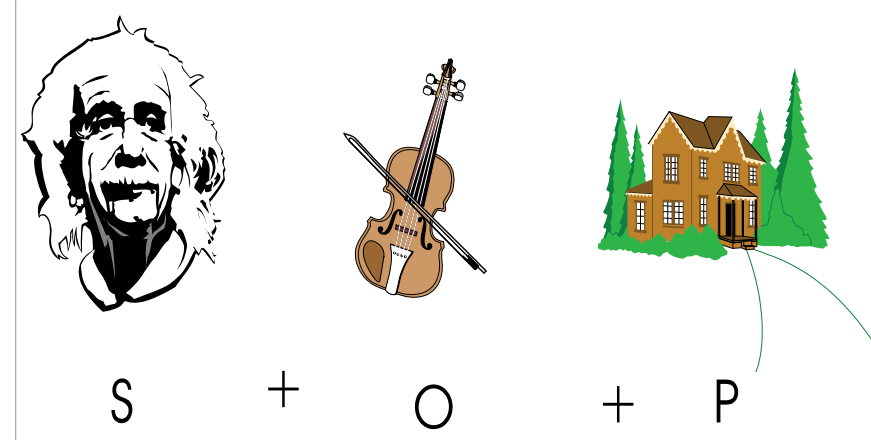

Figure 1: An example of three basic conceptual elements, subject, object and place involved in a story. 
PIN-code in a cued recall test of assigned codes; in Experiment 2, the PIP-code was compared with PIN-code in a recognition test of assigned codes and a backward recall of the cue.

\section{Experiment 1}

In Experiment 1, the recall method was used to measure the performance of assigned PIP- and PIN-codes and to compare the performance variation between these codes. This notion was based on the assumption that sometimes users may have to remember an assigned Personal Identification Code to obtain access to social utilities (e.g. bank, petrol, clearance, smartphone, computer). Thus, twelve names of service systems were prepared as common examples of users' assigned codes in a real life situation.

\section{Method}

\section{Subjects}

Subjects were 56 voluntary undergraduates at Luleå University of Technology and Medical College in Boden randomly assigned to two conditions, one to remember PIN-codes and the other to remember PIP-codes.

\section{Materials}

The pictures were selected from the software of CorelDRAW, which contained 22,000 clip art pictures in various classifications. The most distinctive feature of all these pictures was their simple construction in colour.

PIP- and PIN-codes were treated similarly with respect to the length of study list and number of items that composed each code. The study list consisted of 12 pairs of items. The pictures were selected from a pool of 82 items. Each item pair consisted of a cue word to public service system (e.g. Bank card, Gasoline card) associated to a target code (PIP- or PIN-code). One half of the study list, the length of target code (PIP- and PIN-code) was three items and the other half four items. Each PIP-code was composed of a sequence of pictures in following order; a face (e.g. Bob Dylan), an object (e.g. guitar), and a place or building (e.g. Eiffel Tower). Each selected picture was used only once in the study list of PIP-codes. The PIN-code was composed in a similar way, three or four random numbers were assigned to each target code. In a cued recall test the subjects had to choose the correct pictures to each PIP-code from the pool of 82 pictures. Half were used in a study list and the other half were the lures. The test order in the booklet were changed for the test.

\section{Design}

The design was a $2 \times 2 \times 2$ mixed design with code type (PIP-code and PIN-code) as a between subjects variable and code length (three items and four items) and a retention interval (immediate and after ten days) as within subjects variable.

\section{Procedure}

The experiment was conducted in three stages, namely; learning (studying), immediate and delayed cue recall testing shown in Figure 2.

The subjects were tested in a classroom. During the study, the subjects were instructed to learn a list of cue words associated to PIPcode (alt. PIN-code) presented on a screen. A training list was shown, before the experimenter presented the whole study list for ten minutes. A cued recall test followed the study. The subjects got a booklet of cue word. They have to write down the correct target codes to each cue word in the study list. In PIP-code condition, to find out the correct pictures to each PIP-code, a list of 82 pictures was presented on the screen. Each picture was assigned a number from 1 to 82 . The subject had to write down the correct picture beside the assigned number on the answer sheet in correct order. In PIN-code condition the subject had to write down the correct code numbers to each cue word. Finally, a retest was administered after ten days in a similar way as before. The only change from the first test was reassign of the number to each picture.

\section{Results and Discussion}

\section{Correct performance}

The critical dependent variables were the correct recalled PIP- and PIN- codes. All relevant means are summarized in Table 1.

The means of recalled target codes were treated with $t$ - test. A significant difference between the PIP- and the PIN-codes was found from both the immediate test, $\mathrm{t}(54)=6.95, \mathrm{p}<0.05$, and the delayed test, $\mathrm{t}(15)=8.41, \mathrm{p}<0.05$. In effect, it meant that the PIP-codes were easier to remember compared to the PIP-codes, irrespective of the retention interval.

Regardless of whether the target codes were composed of pictures or digits, no significant effects of code length were obtained in immediate and delayed recall tests. This meant that the length of code was not as critical as the code type in LTM.

The original data were subjected to repeated-measures of analysis of variance. A significant effect of retention interval was found for both the PIP-codes, $\mathrm{F}(1,6)=6.74, \mathrm{p}<0.05$, and the PIN-codes, $\mathrm{F}(1,9)=21.14$, $\mathrm{p}<0.05$. We found that after two weeks the forgetting rate was nearly the same for both types of codes.

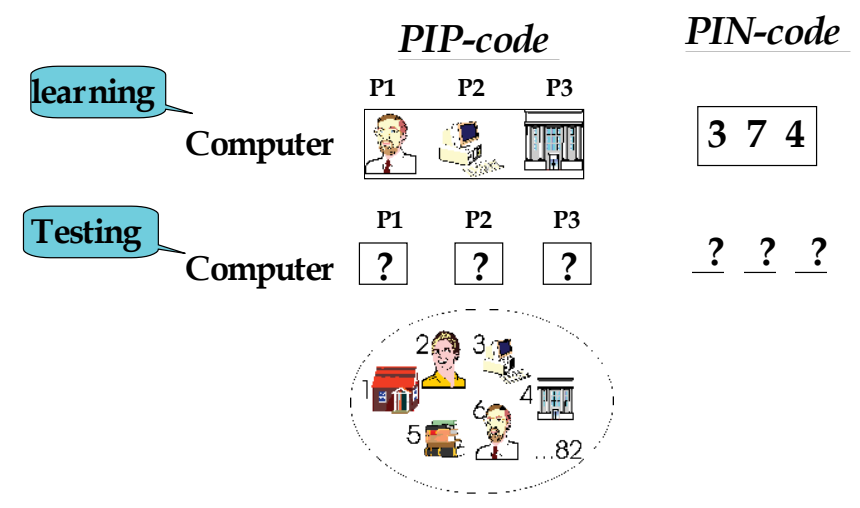

Figure 2: Illustration of the experiment procedure of the cued recall of assigned codes.

\begin{tabular}{|c|c|c|}
\hline Code type & Immediate test & Delayed test \\
\hline PIP-codes & & \\
\hline Three pictures & 4.0 & 2.6 \\
\hline Four pictures & 3.6 & 2.0 \\
\hline Total & 7.6 & 4.6 \\
\hline PIN-codes & & \\
\hline Three digits & 2.4 & 0.5 \\
\hline Four digits & 1.4 & 0.6 \\
\hline Total & 3.8 & 1.1 \\
\hline
\end{tabular}

Table 1: Mean comparison of the correct number of the individual performance in the immediate test and delayed test, for the study of the assigned PIP-and PINcodes. $(\mathrm{N}=12)$. 


\section{Performance error}

The critical dependent variable was the different kinds of errors made by the subjects in the experiment. The percentage of every type of errors is summarized in Table 2 .

In the PIP-code condition, location errors increased $13.0 \%$ between the two recall tests, immediate and delayed. This result revealed that in the delayed test the subjects had difficulty in connecting the cue words to the PIP-codes which they were to recall though they had successfully done so in the immediate test. The substitution errors also increased but not so quickly as the location errors. However, the transportation errors decreased by $4.6 \%$ in the delayed recall test. This was probably due to the condition that the story-grammar had standardized the code order as SOP/T. In the PIN-code condition, most errors were similar to the PIP-codes, due to subjects complete forgetting, that is, the whole code, though location errors also posed problems. The omission errors were non-existent in two recall tests since the response sheet contained two tables prepared separately for three digit PIN-codes and four digit PIN-codes. The subjects had been reminded, to some extent, by this arrangement. Generally, the data analyzes showed the same degree of consistency; that is, the PIP-codes were easier to remember than the PIN-code, irrespective of the retention interval. Additionally, in solving the location problem, a large portion of procedural error dissipated.

\section{Experiment 2}

In the present experiment the task was to test recognition of the target codes of the PIP- and PIN-codes.

\section{Method}

Subjects: Thirty-six student volunteers from Luleå university of Technology were randomly assigned to the two learning conditions: PIN- and PIP-codes.

\section{Materials, Design and Procedure}

The same materials as used in Experiment 1 were used in this experiment. Each item pair consisted of a cue word and target code. During recognition testing, we mixed 12 target codes from the study list with 12 lures not presented in the study list randomly.

This experiment was conducted in the same way as Experiment 1. In a classroom the experimenter instructed subjects to remember a list of item pairs composed of cue words together with target codes, presented on a screen, shown in Figure 3.

After ten minutes, the subjects were administrated a recognition test of the target codes and backward cue recall of the cue word associated

\begin{tabular}{|c|c|c|c|c|}
\hline & \multicolumn{2}{|c|}{ Immediate test } & \multicolumn{2}{c|}{ Delayed test } \\
\hline Type of errors & PIP-code & PIN-code & PIP-code & PIN-code \\
\hline $\begin{array}{c}\text { Procedural } \\
\text { Wrong location }\end{array}$ & 6.0 & 24.0 & 19.0 & 19.4 \\
\hline Clerical & & & & \\
\hline Transportation & 5.8 & 1.0 & 1.2 & 0.0 \\
\hline Substitution & 4.6 & 3.7 & 8.3 & 2.1 \\
\hline Omission & 3.3 & 0.0 & 3.6 & 0.0 \\
\hline Other & & & & \\
\hline No classification & 17.7 & 39.6 & 34.5 & 68.1 \\
\hline Total & 37.4 & 68.3 & 66.6 & 89.6 \\
\hline
\end{tabular}

Table 2: The percentage of different kinds of errors made in the immediate and delayed recall test, for the study of the assigned PIP- and PIN-code.

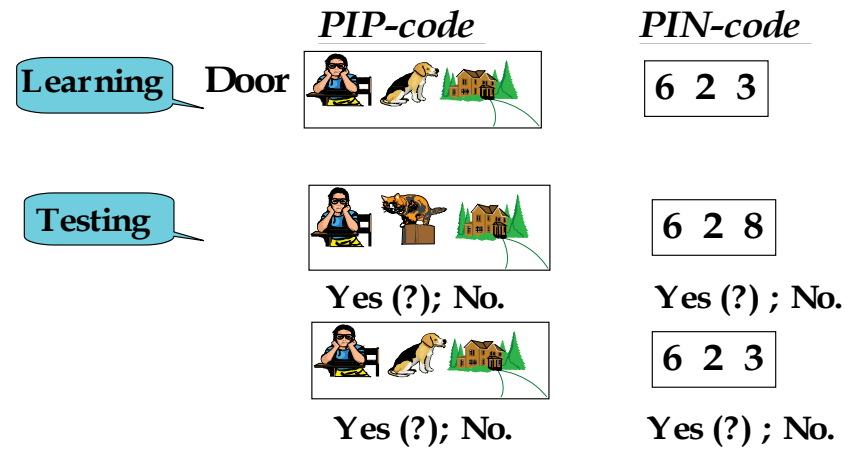

Figure 3: Illustration of the experiment procedure of recognition and cued recall of the assigned codes.

with the target code. In the recognition of PIP-code condition twentyfour items were presented. The task was to recognize every item by writing down "Yes" or "No" on the response sheet, and write down the cue word of the recognized target codes. Finally, two weeks later the test was administered again in a similar way.

\section{Results and Discussion}

\section{Correct performance}

The critical dependent variables were the correct recognized PIP- and PIN-codes and recall of the cue words associated with the recognized codes. All relevant means are summarized in Table 3.

Under the recognition condition, a significant difference was found between the two types of target codes, regardless of the immediate test, $\mathrm{t}(36)=7.73, \mathrm{p}<0.001$, or delayed test, $\mathrm{t}(27)=2.98, \mathrm{p}<0.006$. The significant differences were also found from the immediate test, $t(36)$ $=5.11, \mathrm{p}<0.01$ and the delayed test, $\mathrm{t}(27)=2.32, \mathrm{p}<0.028$. It meant the subjects were very sensitive to different stimuli material. Therefore a significant difference was obtained from the performance of the PIPand PIN-codes, irrespective of the retention interval.

The mean values of correct performance were treated with the t-test. No significant effects of code lengths were obtained, neither in the immediate test nor in the delayed test, in the recognition and recall conditions. Thereby we can conclude that the number of items composition of the target code has no significant effect on recognition of target code or the recall of cues.

Forgetting rate. The data were subjected to a repeated-measures ANOVA. A significant effect of the retention interval for the PIP-codes in the recognition test, $\mathrm{F}(1,47)=20.34, \mathrm{p}<0.001$, and in the recall test, $\mathrm{F}(1,47)=25.35, \mathrm{p}<0.001$, were obtained, but not for the PIN-codes.

\section{Performance error}

One type of performance error pertained to subjects' failure to recall the cue words. Another error correct recall of cue words but associations of these with the wrong target codes (location error). The results are shown in Table 4.

The data demonstrate that the subjects had difficulties in remembering the cue words or to associate them with the target codes even when the name of the code (cue word) was remembered. This performance deteriorated when the subjects were retested after two weeks. The experimental results confirmed that recognition performance exceeds recall. 


\begin{tabular}{|c|c|c|c|c|}
\hline & \multicolumn{2}{|c|}{ Immediate test } & \multicolumn{2}{c|}{ Delayed test } \\
\hline Code type & Recognition & Cued recall & Recognition & Cued recall \\
\hline PIP- codes & & & & \\
\hline Three pictures & 5.8 & 4.9 & 5.0 & 3.2 \\
\hline Four pictures & 5.8 & 4.6 & 4.8 & 2.2 \\
\hline Total & 11.6 & 9.5 & 9.8 & 5.4 \\
\hline PIN- codes & & & & \\
\hline Three pictures & 4.3 & 2.5 & 4.0 & 1.5 \\
\hline Four pictures & 3.9 & 2.0 & 3.0 & 1.3 \\
\hline Total & 8.2 & 4.5 & 7.0 & 2.8 \\
\hline
\end{tabular}

Table 3: Mean comparison of the correct number of the individual performance of the recognized codes as well as recalled cue words in the immediate and delayed test, for the study of assigned PIP- and PIN- codes $(\mathrm{N}=12)$.

\begin{tabular}{|c|c|c|c|c|}
\hline & \multicolumn{2}{|c|}{ Immediate test } & \multicolumn{2}{c|}{ Delayed test } \\
\hline Type of errors & PIP-code & PIN-code & PIP-code & PIN-code \\
\hline Procedural & & & & \\
\hline Wrong location & 3.6 & 16.7 & 11.5 & 22.9 \\
\hline Other & & & & \\
\hline Forgetfulness & 14.3 & 7.5 & 24.2 & 7.3 \\
\hline Total & 17.9 & 24.2 & 35.7 & 30.2 \\
\hline
\end{tabular}

Table 4: The percentage of two kinds of errors made in the immediate and delayed test, for the study of recalling the cue words for the assigned PIP- and PIN-codes.

\begin{tabular}{|c|c|c|}
\hline Code type & Immediate test & Delayed test \\
\hline PIP-codes & $\mathrm{t}(28)=4.16^{* * *}$ & $\mathrm{t}(21)=7.38^{* *}$ \\
\hline PIN-codes & $\mathrm{t}(10)=6.20^{* *}$ & $\mathrm{t}(8)=5.86^{* * *}$ \\
\hline${ }^{* *} \mathfrak{p}<0.01 .{ }^{* * *} \mathrm{p}<0.001$. & & \\
\hline
\end{tabular}

Table 5: Summary of the performance difference between the recognition and recall at the statistics significance level.

The comparison of recognition and recall. A higher performance on recognition than recall was obtained in each condition and each test. The four significant levels are shown in Table 5.

\section{General Discussion}

The results of two experiments support the notion of PIP-codes. The subjects remembered the PIP-codes more effectively than the PINcodes in both experiments. Firstly, using pictures as the target code was the most appropriate material. Secondly, the method improved recall by utilising subjects' semantic and episodic knowledge available and accessible in LTM. Semantic memory maintains information closely tied to language use, widely-shared information; on the other hand, episodic memory stores information more directly related to individual experiences. Thus, in relation to the PIP-codes, recourse to semantic memory implies use of information framed in broad social contexts that facilitate recall. An example is the selection of picture pool, which facilitates comprehensible pictures together with subjects' preferences. Alternatively, recourse to episodic memory implies use of information framed in a limited personal context that may suffice to ensure recall but does not limit the extent of comprehension.

Coherence effects assist memory in encoding and storing the information. Bartlett [4] observed that in the process of remembering a message the first thing the subject tends to recall is his/her attitude towards it: "The recall is then a construction made largely on the basis of his attitude, and its general effect is that of a justification of the attitude" ( $p$ 63). In short, what is remembered is driven to some extent by emotional engagements and response to those events [18,19]. Much evidence suggests that memory for well-integrated associations may be supported by the less-context-specific process of familiarity [20,21]. From the viewpoint of 'meaning-associated memories, Rönnlund et al. [22] have shown that, in a population-based sample of older adults, self-reported memory as assessed by the Prospective and Retrospective Questionnaire may reflect mood-state and personality factors rather than individual differences in memory and cognitive ability which suggests that memory for PIP code ought to hold benefits also for aging populations. Thus, emotional components and their effects upon encoding efficacy ought not to be underestimated.

\section{Memory for PIP code}

The general pattern of the present findings was that a backward recall of the code names was easier than a forward recall of the codes. The occasions where one hears "I forget the number for my account" are probably more frequent than those where one hears "Is this the number for my bank". The first experiment was positive in limit the transportation error, but neglected the location problems by the way to assign the name for every code.

\section{Error Control Alternatives}

The present study makes alternative recommendations for supporting error control. One way is to eliminate the procedural errors by user self-detection, for example, by providing possibilities for users to detect the transportation error with the story-grammar method. Elimination of clerical errors by technique-detection is another, e.g. the ATMs automatically reject a PIP-code when the operation involves location, substitution or omission errors. Generally these two approaches may be applied to the real situations since they involve low cost and effectiveness for the individual. In relation to the present analyses, "MemAxes" uniquely allows users to analyze the different aspects related to memory performance by providing multiple visual contexts for a centralized dataset [23].

\section{Some Limitations of the Study}

In considering the practical implications of these findings, it is important to remember the limitations of the study. First, the study was confined to the group test with the learning material displayed on the screen. This laboratory testing context is not identical to the real life context. Consequently, it is impossible to avoid a bias in the results. Second, the student subjects provide a particular population, characterized by youth and a high educational level, facilitatory for performance. Due to these limitations, caution must be exercised in generalizing the results from the present study to the PIP-code in more natural contexts. The main point of this study is that the memorability of the PIP-code is expected to be robust, dependent on whether the situation requires careful matching of perceptual information against information retrieved from memory.

Nevertheless, the viability of the PIP-code method hinges upon provision of a timely, system-based, "user-friendly" guidance, that addresses remembering and security. Several future directions are available: (i) experiments that test PIP-codes generated by the subjects in comparison with present findings; (ii) construction of standardized picture-bases for research on PIP-codes; and, (iii) tests of the PIP-code model on occupational user groups. It is clear that the low number of participants (Experiment 1: $\mathrm{N}=56$; Experiment 2: $\mathrm{N}=36$ ) provided a low level of power for this study. Nevertheless, in view of the significance levels it would appear that the effects obtained were quite notable [24-27]. 
Citation: Sandberg KW, Pan Y, Archer T (2018) Recall and Recognition of User Codes: PIP versus PIN Personal Identification Code. Clin Exp Psychol 4: 200. doi: 10.4172/2471-2701.1000200

Page 6 of 6

\section{Acknowledgments}

This research was supported by the KFB (KommunikationsForkningsBeredningen), Sweden.

\section{References}

1. Parfit DA (1971) Personal identity. Philosophical Rev 80: 3-27.

2. Wong W (2008) Meaningfulness and identities. Ethical Theory Moral Pract 11: $123-148$.

3. Bailey RW (1989) Human performance engineering. P T R Prentice Hill Englewood Cliffs, New Jersey.

4. Bartlett, Sir Frederick (1932) Remembering. Cambridge University Press, Cambridge, England.

5. Bruce V, Young AW (1986) Understanding face recognition. Br J Psycho 77: 305-327.

6. Bruce V, Young A (2000) In the eye of the beholder: The science of face perception. Oxford: Oxford University Press.

7. Nickerson RS (1965) Short term memory for complex meaningful configurations: A demonstration of captive. Canadian Journal of Psychology 19: $155-160$

8. Shepard RN (1967) Recognition memory for words, sentences and pictures Journal of Verbal Learning and Verbal Behaviour 6: 156-163.

9. Standing L, Conezio J, Haber RN (1970) Perception and memory for pictures: Single trial learning of 2560 visual stimuli. Psychonomic Science 19: 73-74.

10. Barton BF, Barton MS (1984) User-friendly password methods for computermediated information systems. Computers and Security 3: 186-195.

11. Welford AT (1976) Skilled Performance: Perceptual and Motor Skills Glenview, I11: Scott, Foresman and Company.

12. Kirkpatrick EA (1894) An experimental study of memory. Psychological Rev 1: 602-609.

13. Standing L (1973) Learning 10,000 pictures. Q J Exp Pychol 25: 207-222.
14. Goldstein AG, Chance JE (1971) Visual recognition memory for complex configurations. Perception and Psychophysics 9: 237-241.

15. Field MM, Hodge MH, Manley CW, Sonntag L (1971) Guidelines for constructing human performance-based codes. Private Communication.

16. Biederman I (1972) Perceiving real-world scenes. Science 177: 77-80.

17. Tulving E (1972) Episodic and semantic memory. In Tulving E, Donaldson W (Eds.) Organization of memory. Academic Press.

18. Kensinger EA, Garoff-Eaton RJ, Schacter DL (2006) Effects of emotion on memory specificity: memory trade-offs elicited by negative visually arousing stimuli. Journal of Memory and Language 56: 575-591.

19. Murray BD, Kensinger EA (2012) The effects of emotion and encoding strategy on associative memory. Memory and Cognition.

20. Diana RA, Van den Boom W, Yonelinas AP, Ranganath C (2010) ERP correlates of source memory: unitized source information increases familiarity-based retrieval. Brain Res 1367: 278-286.

21. Staresina BP, Davachi L (2010) Object unitization and associative memory formation are supported by distinct brain regions. J Neurosci 30: 9890-9897.

22. Rönnlund M, Vestergren P, Mäntylä T, Nilsson LG (2011) Predictors of self-reported prospective and retrospective memory in a population-based sample of older adults. J Genet Psychol 172: 266-284.

23. Gimenez A, Gamblin T, Jusufi I, Bhatele A, Schulz M, et al. (2018) MemAxes: Visualization and Analytics for Characterizing Complex Memory Performance Behaviors. IEEE Trans Vis Comput Graph 24: 2180-2193.

24. Baddley A (1993) Your memory. Prion, London.

25. Bartlett JC, Leslie JE, Tubbs A, Fulton A (1989) Ageing and memory for pictures of faces. Psychol Aging 4: 276-283.

26. Higbee KL (1977) Your memory - How it works and how to improve it. Prentice-Hall, Inc., New Jersey.

27. Postman L, Kruesi E, Regan J (1975) Recognition and recall as measures of long-term retention. Q J Exp Psychol 27: 411-418. 